C.C. YANG AND B.Q. LI

KODAI MATH. J.

15 (1992), 341-353

\title{
ON THE COMPLETE MEROMORPHIC FUNCTIONS
}

\author{
By Chung Chun Yang and Bao QIN LI
}

\section{Introduction.}

Suppose that $f(z)$ is a non-constant meromorphic function in $|z|<+\infty$. A meromorphic function $a(z)$ is called a small function of $f(z)$ if $T(r, a(z))=$ $o\{T(r, f)\}$ as $r \rightarrow \infty$, we shall call a small function $a(z)$ of $f(z)$ a deficient function of $f(z)$ if and only if

$$
\varliminf_{r \rightarrow \infty} \frac{m\left(r, \frac{1}{f(z)-a(z)}\right)}{T(r, f)}>0 .
$$

$f(z)$ will be called a complete function if it has no deficient function $a(z)$, including $a(z) \equiv \infty$. That is, for any small function $a(z)$ of $f$ and $\infty$, we have

$$
\delta(a(z), f)=\varliminf_{r \rightarrow \infty} \frac{m\left(r, \frac{1}{f-a(z)}\right)}{T(r, f)}=0 \quad \text { and } \quad \delta(\infty, f)=\varliminf_{r \rightarrow \infty} \frac{m(r, f)}{T(r, f)}=0 .
$$

The set of all such complete functions will be denoted by $\tilde{F}$ and the set of all meromorphic functions which assume no deficient functions $a(z)$, except possibly $a(z)$ being identically $\infty$, will be noted by $F$.

The well-known Nevanlinna deficiency relation : $0 \leqq \Sigma \delta(a, f) \leqq 2$, where the sum is taken over all complex numbers $a$, including $\infty$, has been extended to small functions by Steinmetz in [12]. That is,

$$
0 \leqq \Sigma \delta(a(z), 1) \leqq 2
$$

where the sum is taken over all the small functions, including $\infty$. The upper bound 2 is clearly best possible. It is a natural goal to investigate those meromorphic functions $f$ for which the above sum may attain the lower bound 0 , i.e. $f \in \widetilde{F}$. In the case when $f$ is entire, some classes of functions which assume no deficiency function $a(z)$ with $a(z) \not \equiv \infty$, i.e. $f \in F$ (note since $f$ is entire, $\delta(\infty, f)=1$ and so $f \notin \widetilde{F}$.) have been exhibited (For example, see Fuchs [4], Sons $[11], \mathrm{Li}[8,9]$, Li and Dai $[10]$; etc.). Few corresponding results for meromorphic (but not entire) functions have been known. Chuang, Yang and $\mathrm{Yi}$ [2] have attempted to use the properties of differential polynomials of

Received June 24, 1991 , revised December 4, 1991. 
meromorphic functions to consider the case of meromorphic functions. And there they posed the question: If $f_{1}$ and $f_{2} \in \tilde{F}$, does it follow that the product $f_{1} f_{2} \in \widetilde{F}$ ? That is, whether is the space $\widetilde{F}$ closed or not with respect to the common multiplication?

In the present note, following Gol'dberg [5], we will consider the distribution of the arguments of the $a(z)$-points of $f(z)$ (i.e., the zeros of $f(z)-a(z)$ ) for a small function $a(z)$ of $f(z)$ and will prove that some perturbation of the uniformity of the distribution of the arguments of the $a(z)$-points will induct $f(z)$ into the space $\widetilde{F}$ (Theorem 1). Moreover, using Theorem 1 , we then will answer the above question (Theorem 2).

Throughout the paper, we shall adopt the standard notation used in Nevanlinna theory (see e.g. [7], [12]). Moreover, if $f$ and $a(z)$ are meromorphic,

and

$$
\begin{aligned}
& \Theta=\Theta\left(\theta_{1}, \theta_{2}, \cdots, \theta_{n}\right)=\bigcup_{i=1}^{n}\left\{z \mid \arg z=\theta_{i}\right\} \text { denotes a system of rays, } \\
& \omega=\omega(\Theta)=\max \left\{\frac{\pi}{\theta_{\jmath+1}-\theta_{\jmath}} ; 1 \leqq \jmath \leqq n\right\} \quad\left(\theta_{n+1}:=\theta_{1}+2 \pi\right)
\end{aligned}
$$

$$
D(\varepsilon, \Theta)=C-\bigcup_{\imath=1}^{n}\left\{z|| \arg z-\theta_{\jmath} \mid<\varepsilon\right\} \quad(\varepsilon>0),
$$

then $n(r, a(z), \Theta, \varepsilon, f)$ denotes the number of zeros of $f(z)-a(z)$ in the region $\{|z| \leqq r\} \cap D(\varepsilon, \Theta)$. The $a(z)$-points of $f$, i.e. the zeros of $f(z)-a(z)$, are called to be attracted to the system $\Theta$ if for any $\varepsilon>0$,

$$
n(r, a(z), \Theta, \varepsilon, f)=o\{T(r, f)\} \quad \text { as } r \rightarrow \infty .
$$

Also, if $\alpha \geqq 0, \beta \geqq 0,0<\beta-\alpha \leqq 2 \pi, k=\pi / \beta-\alpha$ and $z_{n}=\rho_{n} e^{2 \phi_{n}}$ denotes the poles of $f$ (counted with multiplicity), then we, similarly as defined in [6], set

$$
\begin{aligned}
& A_{\alpha \beta}(r, f)=\frac{k}{\pi} \int_{l}^{r}\left(\frac{r^{k}}{t^{k}}-\frac{t^{k}}{r^{k}}\right)\left(l n^{+}\left|f\left(t e^{\imath \alpha}\right)\right|+l n^{+}\left|f\left(t e^{\imath \beta}\right)\right|\right) \frac{d t}{t}, \\
& B_{\alpha \beta}(r, f)=\frac{2 k}{\pi} \int_{\alpha}^{\beta} l n^{+}\left|f\left(r e^{\imath \phi}\right)\right| \sin k(\phi-\alpha) d \phi, \\
& C_{\alpha \beta}(r, f)=2 k \int_{1}^{r}\left(\sum_{\substack{1 \leqq \rho_{n} \leqq t \\
\alpha \leqq \phi}} \sin k\left(\phi_{n}-\alpha\right)\right)\left(\frac{r^{k}}{t^{k}}+\frac{t^{k}}{r^{k}}\right) \frac{d t}{t}, \\
& S_{\alpha \beta}(r, f)=A_{\alpha \beta}(r, f)+B_{\alpha \beta}(r, f)+C_{\alpha \beta}(r, f) .
\end{aligned}
$$

We define that $S_{\alpha \beta}(r, f=a(z))=S_{\alpha \beta}(r, 1 /(f-a(z)))$. Similarily, we can define $A_{\alpha \beta}(r, f=a(z)), B_{\alpha \beta}(r, f=a(z))$ and $C_{\alpha \beta}(r, f=a(z))$. Recall the Valiron deficiency

$$
\Delta(a(z), f)=\varlimsup_{r \rightarrow \infty} \frac{m\left(r, \frac{1}{f-a(z)}\right)}{T(r, f)}=1-\varliminf_{r \rightarrow \infty} \frac{N\left(r, \frac{1}{f-a(z)}\right)}{T(r, f)} .
$$


THEOREM 1. Suppose that $\Theta$ is some system of rays and $f$ is a meromorphic function of finite order $\lambda>\omega$. If $\Delta(b(z), f)=0$ and $b(z)$-points of $f$ are attracted to $\Theta$ for a small function $b(z)(b(z)$ can be $\infty)$, then $\delta(a(z), f)=0$ for any small function $a(z)$, including $\infty$. That is, $f \in \widetilde{F}$.

THEOREM 2. There exist two functions $f_{1} \in \tilde{F}$ and $f_{2} \in \tilde{F}$ such that $f_{1} f_{2} \notin \tilde{F}$.

That is, the space $\tilde{F}$ is not closed w.r.t. the common multiplication.

Finally in Pan 5, we will construct a class of meromorphic functions in $\tilde{F}$ which may be of infinite orders.

\section{Lemmas.}

In order to prove our theorems, we need some lemmas as follows.

LEMMA 1 [10]. Suppose that $f(z)$ is a meromorphic function such that for some large $R$ and some $\lambda(\geqq 1), T(R, f)<R^{\lambda}$.

Let $n$ be an arbitrary positive integer. Then there exists a set $E$ satisfying ln mes $(E \cap[1, R]) \geqq(1-1 / n) \ln R+O(1)$ as $R \rightarrow \infty$ such that for $r \in E, \ln M(r, f)$ $\leqq c \lambda^{2} n^{4} T(r, f)$, where $c$ is an absolute constant.

LEMMA 2 [6]. Let $f(z)$ be a meromorphic function, $k>1,0<\delta \leqq 2 \pi$ and $r \geqq 1$. Then for any measurable set $E_{r} \subset[0,2 \pi]$ with mes $E_{r}=\delta$, we have that

$$
\int_{E_{r}} \ln \left|f\left(r e^{\imath \phi}\right)\right| d \phi \leqq \frac{6 k}{k-1} \delta\left(\ln \frac{2 \pi e}{\delta}\right) T(k r, f) .
$$

LEMMA 3 [6]. Let $f(z)$ be a non-constant meromorphic function in the sector $\{z \mid \alpha \leqq \arg z \leqq \beta\}(\alpha \geqq 0, \beta \geqq 0,0<\beta-\alpha \leqq 2 \pi)$. Then for any complex number $a \neq \infty$,

$$
S_{\alpha \beta}(r, f=a)=S_{\alpha \beta}(r, f)+O(1) r^{k} \text { as } r \rightarrow \infty \text {, where } k=\pi /(\beta-\alpha) \text {. }
$$

LEMMA 4. Let $f(z)$ be a non-constant meromorphic function. Then for any two small functions $a(z), b(z)$ we have

$$
S_{\alpha \beta}(r, f=a(z)) \leqq S_{\alpha \beta}(r, f=b(z))+S_{\alpha \beta}(r, b(z)-a(z))+O(1) r^{k} \text { as } r \rightarrow \infty,
$$

where $0<\beta-\alpha \leqq 2 \pi$ and $k=\pi /(\beta-\alpha)$.

Proof. By Lemma 3,

$$
\begin{aligned}
S_{\alpha \beta}(r, f=a(z)) & =S_{\alpha \beta}\left(r, \frac{1}{f-a(z)}\right) \\
& =S_{\alpha \beta}(r, f-a(z))+O(1) r^{k} \\
& =A_{\alpha \beta}(r, f-a(z))+B_{\alpha \beta}(r, f-a(z))+C_{\alpha \beta}(r, f-a(z))+O(1) r^{k} \text { (see (5)). }
\end{aligned}
$$

Also by (2), (3) and (4), we can easily deduce that 


$$
\begin{aligned}
& A_{\alpha \beta}(r, f-a(z)) \\
& =\frac{k}{\pi} \int_{1}^{r}\left(\frac{r^{k}}{t^{k}}-\frac{t^{k}}{r^{k}}\right)\left(\ln n^{+}\left|f\left(t e^{\imath \alpha}\right)-a\left(t e^{2 \alpha}\right)\right|+\ln +\left|f\left(t e^{\imath \beta}\right)-a\left(t e^{\imath \beta}\right)\right|\right) \frac{d t}{t} \\
& \leqq \frac{k}{\pi} \int_{1}^{r}\left(\frac{r^{k}}{t^{k}}-\frac{t^{k}}{r^{k}}\right)\left(\ln n^{+}\left|f\left(t e^{\imath \alpha}\right)-b\left(t e^{2 \alpha}\right)\right|+\ln n^{+}\left|b\left(t e^{\imath \alpha}\right)-a\left(t e^{\imath \alpha}\right)\right|+\ln 2\right. \\
& \left.+\ln n^{+}\left|f\left(t e^{\imath \beta}\right)-b\left(t e^{2 \beta}\right)\right|+\ln ^{+}\left|b\left(t e^{\imath \beta}\right)-a\left(t e^{\imath \beta}\right)\right|+\ln 2\right) \frac{d t}{t} \\
& \leqq A_{\alpha \beta}(r, f-b(z))+A_{\alpha \beta}(r, b(z)-a(z))+\frac{2 k \ln 2}{\pi} \int_{1}^{r} \frac{r^{k}}{t^{k+1}} d t \\
& \leqq A_{\alpha \beta}(r, f-b(z))+A_{\alpha \beta}(r, b(z)-a(z))+O(1) r^{k} \text {, } \\
& B_{\alpha \beta}(r, f-a(z)) \\
& =\frac{2 k}{\pi} \int_{\alpha}^{\beta} l n^{+}\left|f\left(r e^{2 \phi}\right)-a\left(r e^{\imath \phi}\right)\right| \sin k(\phi-\alpha) d \phi \\
& \leqq \frac{2 k}{\pi} \int_{\alpha}^{\beta}\left(l n^{+}\left|f\left(r e^{\imath \phi}\right)-b\left(r e^{\imath \phi}\right)\right|+l n^{+}\left|b\left(r e^{\imath \phi}\right)-a\left(r e^{\imath \phi}\right)\right|+\ln 2\right) \sin k(\phi-\alpha) d \phi \\
& \leqq B_{\alpha \beta}(r, f-b(z))+B_{\alpha \beta}(r, b(z)-a(z))+O(1),
\end{aligned}
$$

and

$$
C_{\alpha \beta}(r, f-a(z))=2 k \int_{1}^{r}\left(\sum_{\substack{1 \leq \rho_{n} \leq t \\ \alpha \leqq \phi_{n} \leqq \beta}} \sin k\left(\phi_{n}-\alpha\right)\right)\left(\frac{r^{k}}{t^{k}}+\frac{t^{k}}{r^{k}}\right) \frac{d t}{t},
$$

where $\rho_{n} e^{\imath \phi_{n}}$ are the poles of $f(z)-a(z)$ (counted with multiplicity). Suppose that $\left\{\boldsymbol{\rho}_{n}^{\prime} e^{\imath \phi_{n}^{\prime}}\right\}$ and $\left\{\boldsymbol{\rho}_{n}^{\prime \prime} e^{\imath \phi_{n}^{\prime \prime}}\right\}$ are the sets of the poles of $f(z)-b(z)$ and $b(z)-a(z)$ (counted with multiplicity), respectively. Then obviously we have that $\left\{\rho_{n} e^{\jmath \phi_{n}}\right\}$ $\subset\left\{\rho_{n}^{\prime} e^{\imath \phi_{n}^{\prime}}\right\} \cup\left\{\rho_{n}^{\prime \prime} e^{\imath \phi_{n}^{\prime \prime}}\right\}$. Hence

$$
\begin{aligned}
C_{\alpha \beta}(r, f-a(z)) & \leqq 2 k \int_{1}^{r}\left(\sum_{\substack{1 \leq \rho_{n}^{\prime} \leq t \\
\alpha \leqq \phi_{n}^{\prime} \leqq \beta}} \sin k\left(\phi_{n}^{\prime}-\alpha\right)+\sum_{\substack{1 \leq \rho_{n}^{\prime \prime} \leq t \\
\alpha \leqq \phi_{n}^{\prime \prime} \leqq \beta}} \sin k\left(\phi_{n}^{\prime \prime}-\alpha\right)\right)\left(\frac{r^{k}}{t^{k}}+\frac{t^{k}}{r^{k}}\right) \frac{d t}{t} \\
& =C_{\alpha \beta}(r, f-b(z))+C_{\alpha \beta}(r, b(z)-a(z)) .
\end{aligned}
$$

Now from the above, we obtain that

$$
S_{\alpha \beta}(r, f=a(z)) \leqq S_{\alpha \beta}(r, f=b(z))+S_{\alpha \beta}(r, b(z)-a(z))+O(1) r^{k} .
$$

LEMMA 5. Suppose that $f(z)$ is meromorphic function of finite order $\lambda>0$, then for any $\rho, 0<\rho<\lambda$, there must be a sequence $\left\{r_{j}\right\} \rightarrow \infty$ as $j \rightarrow \infty$ and a $r_{0}>0$ such that for $r_{0} \leqq t \leqq r_{j}(j=1,2,3, \cdots)$,

$$
\frac{T(t, f)}{T\left(r_{\jmath}, f\right)} \leqq\left(\frac{t}{r_{\jmath}}\right)^{\rho},
$$


and

$$
\left.T\left(2 r_{\jmath}, f\right) \leqq 2^{\lambda+2} T\left(r_{\jmath}, f\right) \quad \text { for large }\right] \text {, }
$$

$$
T\left(r_{\jmath}, f\right) r_{j}^{-\rho} \longrightarrow \infty \quad \text { as } j \rightarrow \infty \text {. }
$$

Moreover, if $g(z)$ is a small function of $f$, then

$$
S_{a \beta}\left(r_{\jmath}, g(z)\right)=A_{\alpha \beta}\left(r_{\jmath}, g(z)\right)+o\left\{T\left(r_{\jmath}, f\right)\right\}, \quad \text { as } r \rightarrow \infty,
$$

where $\alpha \geqq 0, \beta \geqq 0,0<\beta-\alpha<2 \pi$ and $k=\pi / \beta-\alpha<\rho$.

Proof. Since $f(z)$ is of finite order $\lambda>0$, it must have a proximate order $\lambda(r)$ (see [3] or [12]) which is real, continuous, and piecewisely differentiable for $r \geqq 1$ having the following properties :

(a) $\lim _{r \rightarrow \infty} \lambda(r)=\lambda$

(b) $\lim _{r \rightarrow \infty} r \lambda^{\prime}(r) \log r=0$

(c) $r^{\lambda(r)} \geqq T(r, f)$ for large $r$ and there is a sequence $\left\{r_{j}\right\} \rightarrow \infty$ such that $r_{j}^{\lambda\left(r_{j}\right)}=T\left(r_{j}, f\right)$. It's easy to verify that $r^{\lambda(r)} r^{-\rho}$ is increasing for $r \geqq r_{0}^{\prime} \geqq 1$ by (a) and (b). Therefore, in view of (c),

$$
T(t, f) t^{-\rho} \leqq t^{\lambda(t)} t^{-\rho} \leqq r_{j}^{\lambda\left(r_{j}\right)} r_{j}^{-\rho}=T\left(r_{\jmath}, f\right) r_{j}^{-\rho} \quad \text { for } \quad r_{0}^{\prime \prime} \leqq t \leqq r_{\jmath},
$$

i.e. (6) holds by setting $r_{0}=\max \left(r_{0}^{\prime}, r_{0}^{\prime \prime}\right)$. Again by (c),

$$
T\left(r_{j}, f\right) r_{j}^{-\rho}=r_{j}^{\lambda\left(r_{j}\right)-\rho} \longrightarrow \infty \quad \text { since } \lambda\left(r_{j}\right) \rightarrow \lambda>\rho .
$$

Now taking small $\varepsilon$ and large $r_{\jmath}$,

$$
T\left(2 r_{\jmath}, f\right) \leqq\left(2 r_{j}\right)^{\lambda\left(2 r_{j}\right)}=2^{\lambda\left(2 r_{j}\right)} r_{j}^{\lambda\left(2 r_{j}\right)}=2^{\lambda+1} r_{j}^{\lambda\left(r_{j}\right)+\varepsilon} \leqq 2^{\lambda+1} 2 r_{j}^{\lambda\left(r_{j}\right)}=2^{\lambda+2} T\left(r_{j}, f\right) .
$$

That is, (7) holds. Next, if $g(z)$ is a small function of $f$, then

$$
\begin{array}{r}
S_{\alpha \beta}\left(r_{\jmath}, g(z)\right)=A_{\alpha \beta}\left(r_{\jmath}, g(z)\right)+B_{\alpha \beta}\left(r_{\jmath}, g(z)\right)+C_{\alpha \beta}\left(r_{\jmath}, g(z)\right), \\
B_{\alpha \beta}\left(r_{\jmath}, g(z)\right)=\frac{2 k}{\pi} \int_{\alpha}^{\beta} l n^{+}\left|g\left(r_{j} e^{2 \phi}\right)\right| \sin k(\phi-\alpha) d \phi \\
\leqq 4 k \frac{1}{2 \pi} \int_{0}^{2 \pi} \ln \left|g\left(r_{j} e^{2 \phi}\right)\right| d \phi \\
=4 k m\left(r_{\jmath}, g\right) \leqq 4 k T\left(r_{\jmath}, g\right)=o\left\{T\left(r_{\jmath}, f\right)\right\},
\end{array}
$$

and

$$
C_{\alpha \beta}\left(r_{j}, g(z)\right)=2 k \int_{1}^{r}\left(\sum_{\substack{1 \leq \rho_{n} \leq t \\ \alpha \leq \phi_{n} \leq \beta}} \sin k\left(\phi_{n}-\alpha\right)\right)\left(\frac{r_{j}^{k}}{t^{k}}+\frac{t^{k}}{r_{j}^{k}}\right) \frac{d t}{t},
$$

where $\rho_{n} e^{\imath \phi_{n}}$ are the poles of $g(z)$ (counted with multiplicity). Hence

$$
C_{\alpha \beta}\left(r_{\jmath}, g(z)\right) \leqq 4 k \int_{1}^{r_{\jmath}} n(t, g(z)) \frac{r_{j}^{k}}{t^{k}} \frac{d t}{t}
$$




$$
\begin{aligned}
& =4 k r_{j}^{k} \int_{1}^{r_{\jmath}} \frac{n(t, g(z))}{t^{k+1}} d t \\
& =4 k r_{j}^{k} \int_{1}^{r_{\jmath}} \frac{1}{t^{k}} d N(t, g(z)) \\
& =4 k r_{\jmath}^{k}\left[\frac{N\left(r_{\jmath}, g\right)}{r_{\jmath}^{k}}-\frac{N(1, g)}{1^{k}}+k \int_{1}^{r} \frac{N(t, g)}{t^{k+1}} d t\right] \\
& \leqq 4 k N\left(r_{\jmath}, g\right)+4 k^{2} r_{j}^{k} \int_{1}^{r_{j}} \frac{T(t, g)}{t^{k+1}} d t \\
& \leqq 4 k T\left(r_{\jmath}, g\right)+4 k^{2} r_{j}^{k} o(1) \int_{1}^{r_{\jmath}} T(t, f) t^{-\rho} t^{\rho-k-1} d t \\
& \left.\leqq 4 k T\left(r_{\jmath}, g\right)+4 k^{2} r_{j}^{k} o(1) T\left(r_{\jmath}, f\right) r_{j}^{-\rho} \int_{r_{0}}^{r_{\jmath}} t^{\rho-k-1} d t+O(1) r^{k} \quad \text { (see }(6)\right) \\
& \leqq o\left\{T\left(r_{\jmath}, f\right)\right\}+4 k^{2} o(1) T\left(r_{\jmath}, f\right) r_{\jmath}^{k-\rho} \frac{1}{\rho-k}\left(r_{j}^{\rho-k}-r_{0}^{\rho-k}\right)(\text { since } k<\rho<\lambda) \\
& =o\left\{T\left(r_{\jmath}, f\right)\right\} \quad \text { as } \quad j \rightarrow \infty .
\end{aligned}
$$

Tnus by (10), (11), (12), we deduce that

$$
S_{\alpha \beta}\left(r_{\jmath}, g\right)=A_{\alpha \beta}\left(r_{\jmath}, g\right)+o\left\{T\left(r_{\jmath}, f\right)\right\}, \quad \text { as } \quad r \rightarrow \infty .
$$

This proves that (9) holds.

LEMMA 6. Suppose that $f(z)$ is a meromorphic function satisfying, for $1 \leqq t$ $\leqq r, \max \left\{T(t, f) t^{-\rho}\right\}=O\left\{T(r, f) r^{-\rho}\right\}$ for some $\rho>0$ and that $g(z)$ is a small function of $f$. Then $S_{\alpha \beta}(r, g)=A_{\alpha \beta}(r, g)+o\{T(r, f)\}$ as $r \rightarrow \infty$, where $\alpha \geqq 0, \beta \geqq 0$, and $k=\pi /(\beta-\alpha)<\rho$.

Proof. By the hypotheses, there exists a $M>0$ such that $T(t, f) t^{-\rho} \leqq$ $M T(r, f) r^{-\rho}$, i.e. $T(t, f) / T(r, f) \leqq M(t / r)^{\rho}$ for $1 \leqq t \leqq r$. Recall when we proved (9) in Lemma 5 we only needed the hypothesis (6). Thus by the same way as in Lemma 5, we can prove the result of this lemma. We omit the details here.

\section{The Proofs of Theorem 1 and Theorem 2.}

Proof of Theorem 1. In the following, we can assume that $a(z) \not \equiv \infty$ and $b(z) \not \equiv \infty$, only for not making the expression ambiguous. For example, if $a(z) \equiv \infty$, we only need to consider

$$
\delta(\infty, f)=\lim _{r \rightarrow \infty} \frac{m(r, f)}{T(r, f)} \text { in place of } \delta(a(z), f)=\lim _{r \rightarrow \infty} \frac{m\left(r, \frac{1}{f-a(z)}\right)}{T(r, f)} .
$$


Also, without loss of generality, we can assume that the system $\Theta$ only consists of one ray $\Theta=\{z \mid \arg z=0\}$ since in the general case we can consider each sector $\left\{z \mid \theta_{\jmath} \leqq \arg z \leqq \theta_{\jmath+1}\right\}$ in the same manner as follows.

Let $n$ be a large positive integer and $\alpha \in[1 / n, 2 / n]$. Then by Lemma 4 with $\alpha$ and $\beta=2 \pi-\alpha$, we have that

$$
\begin{aligned}
B_{\alpha \beta}(r, f=a(z)) & \leqq S_{\alpha \beta}(r, f=a(z)) \\
& \leqq S_{\alpha \beta}(r, f=b(z))+S_{\alpha \beta}(r, b(z)-a(z))+O(1) r^{k} \quad \text { as } r \rightarrow \infty,
\end{aligned}
$$

where $k=\pi / \beta-\alpha=\pi / 2 \pi-2 \alpha=\pi / 2(\pi-2 / n)$. Since $\lambda>\omega=1 / 2$, we can assume $n$ so large that $k<\lambda$.

Taking $\rho \in(k, \lambda)$ and using Lemma 5 with $g(z)=b(z)-a(z)$, we can find a sequence $\left\{r_{j}\right\} \rightarrow \infty$ such that (6), (7), (8), (9) all holds. Now by (9),

$$
S_{\alpha \beta}\left(r_{\jmath}, b(z)-a(z)\right)=A_{\alpha \beta}\left(r_{\jmath}, b(z)-a(z)\right)+o\left\{T\left(r_{\jmath}, f\right)\right\}, \quad \text { as } \quad r \rightarrow \infty .
$$

By the definition of $S_{\alpha \beta}(r, f=b(z))$, we have that

$$
\begin{aligned}
S_{\alpha \beta}\left(r_{\jmath}, f=b(z)\right)= & A_{\alpha \beta}\left(r_{\jmath}, f=b(z)\right)+B_{\alpha \beta}\left(r_{\jmath}, f=b(z)\right)+C_{\alpha \beta}\left(r_{\jmath}, f=b(z)\right) \\
= & A_{\alpha \beta}\left(r_{\jmath}, f=b(z)\right)+\frac{2 k}{\pi} \int_{\alpha}^{2 \pi-\alpha} l n^{+}\left|\frac{1}{f\left(r_{j} e^{2 \phi}\right)-b\left(r_{j} e^{2 \phi}\right)}\right| \sin k(\phi-\alpha) d \phi \\
& +2 k \int_{1}^{r_{\jmath}}\left(\sum_{\substack{1 \leq \rho_{n} \leqq t \\
\alpha \leqq \phi_{n} \leqq \beta}} \sin k\left(\phi_{n}-\alpha\right)\right)\left(\frac{r_{j}^{k}}{t^{k}}+\frac{t^{k}}{r_{j}^{k}}\right) \frac{d t}{t},
\end{aligned}
$$

where $\rho_{n} e^{\imath \phi_{n}}$ are zeros of $f-b(z)$ (counted with multiplicity). It's clear that, in view of (6),

$$
\begin{aligned}
\int_{r_{0}}^{r_{\jmath}} T(t, f) \frac{r_{j}^{k}}{t^{k+1}} d t & =r_{j}^{k} \int_{r_{0}}^{r_{\jmath}} T(t, f) t^{-\rho} t^{\rho-k-1} d t \\
& \leqq r_{j}^{k} \int_{r_{0}}^{r_{\jmath}} T\left(r_{\jmath}, f\right) r_{j}^{-\rho} t^{\rho-k-1} d t \\
& \leqq \frac{1}{\rho-k} T\left(r_{\jmath}, f\right) .
\end{aligned}
$$

Thus by (15), we have that

$$
\begin{aligned}
& S_{\alpha \beta}\left(r_{\jmath}, f=b(z)\right) \\
\leqq & A_{\alpha \beta}\left(r_{\jmath}, f=b(z)\right)+4 k m\left(r_{\jmath}, \frac{1}{f-b(z)}\right)+4 k \int_{1}^{r_{\jmath}} n\left(t, b(z), \Theta, \frac{1}{n}, f\right) \frac{r_{j}^{k}}{t^{k}} \frac{d t}{t} \\
\leqq & A_{\alpha \beta}\left(r_{\jmath}, f=b(z)\right)+o\left\{T\left(r_{\jmath}, f\right)\right\}+o(1) \int_{r_{0}}^{r_{\jmath}} T(t, f) \frac{r_{\jmath}^{k}}{t^{k+1}} d t \\
\leqq & A_{\alpha \beta}\left(r_{\jmath}, f=b(z)\right)+o\left\{T\left(r_{\jmath}, f\right)\right\}, \quad \text { (by the hypotheses and (1)) }
\end{aligned}
$$


Now combining (17), (14) with (13), we obtain that, in view of (8),

$$
\begin{aligned}
B_{\alpha \beta}\left(r_{\jmath}, f=a(z)\right) & \leqq A_{\alpha \beta}\left(r_{\jmath}, f=b(z)\right)+A_{\alpha \beta}\left(r_{\jmath}, b(z)-a(z)\right)+o\left\{T\left(r_{\jmath}, f\right)\right\}+O(1) r_{\jmath}^{k} \\
& \leqq A_{\alpha \beta}\left(r_{\jmath}, f=b(z)\right)+A_{\alpha \beta}\left(r_{\jmath}, b(z)-a(z)\right)+o\left\{T\left(r_{\jmath}, f\right)\right\}
\end{aligned}
$$

But

$$
\begin{aligned}
B_{\alpha \beta}\left(r_{\jmath}, f=a(z)\right) & =\frac{2 k}{\pi} \int_{\alpha}^{\beta} \ln \left|\frac{1}{f\left(r_{j} e^{2 \phi}\right)-a\left(r_{j} e^{2 \phi}\right)}\right| \sin k(\phi-\alpha) d \phi \\
& \geqq \frac{2 k}{\pi} \int_{4 / n}^{2 \pi-4 / n} \ln ^{+}\left|\frac{1}{f\left(r_{j} e^{2 \phi}\right)-a\left(r_{j} e^{2 \phi}\right)}\right| \sin k(\phi-\alpha) d \phi
\end{aligned}
$$

Notice that

$$
\begin{aligned}
k(\phi-\alpha) & =\frac{\pi}{2 \pi-2 \alpha}(\phi-\alpha) \\
& \leqq \frac{\pi}{2 \pi-4 / n}\left(2 \pi-\frac{5}{n}\right) \\
& =\pi\left\{1-\frac{1}{n(2 \pi-4 / n)}\right\} \leqq \pi-\frac{1}{2 n}
\end{aligned}
$$

and

$$
k(\phi-\alpha) \geqq \frac{\pi}{2 \pi}\left(\frac{4}{n}-\frac{2}{n}\right)=\frac{1}{n} \geqq \frac{1}{2 n}
$$

provided that $4 / n \leqq \phi \leqq 2 \pi-4 / n$. We thus have $\sin k(\phi-\alpha) \geqq \sin 1 / 2 n$ and so that

$$
B_{\alpha \beta}\left(r_{\jmath}, a=b(z)\right) \geqq \frac{2 k}{\pi} \int_{4 / n}^{2 \pi-4 / n} l n^{+}\left|\frac{1}{f\left(r_{j} e^{2 \phi}\right)-a\left(r_{j} e^{2 \phi}\right)}\right| \sin \frac{1}{2 n} d \phi .
$$

We deduce that, by (18),

$$
\begin{aligned}
& \int_{4 / n}^{2 \pi-4 / n} \ln +\frac{1}{\left|f\left(r_{j} e^{2 \phi}\right)-a\left(r_{j} e^{2 \phi}\right)\right|} d \phi \\
\leqq & \frac{\pi}{2 k \sin 1 / 2 n}\left(A_{\alpha \beta}\left(r_{\jmath}, f=b(z)\right)+A_{\alpha \beta}\left(r_{\jmath}, b(z)-a(z)\right)+o\left\{T\left(r_{\jmath}, f\right)\right\} .\right.
\end{aligned}
$$

Integrating (19) for $\alpha \in[1 / n, 2 / n]$, we have that

$$
\begin{aligned}
& \frac{1}{n} \int_{4 / n}^{2 \pi-4 / n} \ln \frac{1}{\left|f\left(r_{j} e^{2 \phi}\right)-a\left(r_{j} e^{2 \phi}\right)\right|} d \phi \\
\leqq & \frac{\pi}{2 k \sin 1 / 2 n}\left(\int_{1 / n}^{2 / n} A_{\alpha \beta}\left(r_{\jmath}, f=b(z)\right) d \alpha+\int_{1 / n}^{2 / n} A_{\alpha \beta}\left(r_{\jmath}, b(z)-a(z)\right) d \alpha\right)+o\left\{T\left(r_{j}, f\right)\right\} .
\end{aligned}
$$

Obviously,

$$
\int_{1 / n}^{2 / n} A_{\alpha \beta}\left(r_{3}, f=b(z)\right) d \alpha \leqq \frac{k}{\pi} \int_{1}^{r_{J}}\left(\frac{r_{J}^{k}}{t^{k}}-\frac{t^{k}}{r_{J}^{k}}\right) \frac{d t}{t} \int_{-2 / n}^{2 / n} l n^{+} \frac{1}{\left|f\left(t e^{2 \alpha}\right)-b\left(t e^{2 \alpha}\right)\right|} d \alpha
$$




$$
\begin{aligned}
& \leqq 2 k \int_{1}^{r_{\jmath}}\left(\frac{r_{J}^{k}}{t^{k}}-\frac{t^{k}}{r_{J}^{k}}\right) m\left(t, \frac{1}{f-b(z)}\right) \frac{d t}{t} \\
& \leqq 2 k o(1) \int_{1}^{r_{\jmath}} T(t, f) \frac{r_{j}^{k}}{t^{k}} \frac{d t}{t} \quad \text { (by the hypotheses) } \\
& =o\left\{T\left(r_{\jmath}, f\right)\right\} \quad \text { as } \quad r_{j} \rightarrow \infty \quad \text { (by (16)). }
\end{aligned}
$$

With the same reason,

$$
\int_{1 / n}^{2 / n} A_{\alpha \beta}\left(r_{\jmath}, b(z)-a(z)\right) d \alpha=\curvearrowleft\left\{T\left(r_{\jmath}, f\right)\right\} \quad \text { as } \quad \jmath \rightarrow \infty .
$$

Therefore, we have proved that

$$
\int_{4 / n}^{2 \pi-4 / n} \ln +\frac{1}{\left|f\left(r_{j} e^{\imath \phi}\right)-a\left(r_{j} e^{\imath \phi}\right)\right|} d \phi=o\left\{T\left(r_{j}, f\right)\right\} \quad \text { as } \quad r_{j} \rightarrow \infty .
$$

On the other hand, using Lemma 2, we have that, in view of (7),

$$
\int_{-4 / n}^{4 / n} \ln +\frac{1}{\left|f\left(r_{j} e^{\imath \phi}\right)-a\left(r_{j} e^{\imath \phi}\right)\right|} d \phi \leqq C_{\lambda} \frac{\ln n}{n} T\left(r_{\jmath}, f\right),
$$

where $C_{\lambda}$ is a constant only depending on $\lambda$. Hence by (21) and (20) we have that

$$
m\left(r_{\jmath}, \frac{1}{f-a(z)}\right) \leqq C_{\lambda} \frac{\ln n}{n} T\left(r_{\jmath}, f\right)+o\left\{T\left(r_{\jmath}, f\right)\right\} .
$$

But $n$ can be assumed arbitrarily large, thus we conclude that

$$
\delta(a(z), f)=\lim _{r \rightarrow \infty} \frac{m\left(r, \frac{1}{f(z)-a(z)}\right)}{T(r, f)}=0 .
$$

This also completes the proof of Theorem 1 .

Proof of Theorem 2. Suppose that $f$ is a meromorphic function satisfying the hypotheses of the Theorem 1 (such functions exist, see Remark 1). That is, $f$ is a meromorphic function of finite order $\lambda>\omega$ for some system $\Theta$ of rays such that $\Delta(b(z), f)=0$ and $n(r, b(z), \Theta, \varepsilon, f)=o\{T(r, f)\}$ for some small function $b(z)$. Let's set

$$
f_{1}(z)=f(z)-b(z) \text { and } f_{2}(z)=\frac{a(z)}{f_{1}(z)},
$$

where $a(z)(\not \equiv 1)$ is an arbitrary entire small function of $f$. Then clearly, $f_{1}$ and $f_{2}$ are of order $\lambda, \Delta\left(0, f_{1}\right)=0, \Delta\left(\infty, f_{2}\right)=0, n\left(r, 0, \Theta, \varepsilon, f_{1}\right)=o\left\{T\left(r, f_{1}\right)\right\}$, and $n\left(r, \infty, \Theta, \varepsilon, f_{2}\right)=o\left\{T\left(r, f_{2}\right)\right\}$. That is, $f_{1}$ and $f_{2}$ satisfy the hypotheses of Theorem 1. Thus, by the result of Theorem $1, f_{1} \in \widetilde{F}$ and $f_{2} \in \widetilde{F}$. But $f_{1} f_{2}=$ $a(z) \notin \tilde{F}$ since $\delta(\infty, a(z))=1$.

In addition, if we assume $a(z)$ to be transcendental, then we will have the 
result: there are two functions $f_{1} \in \tilde{F}$ and $f_{2} \in \tilde{F}$ such that $f_{1} f_{2}$ is transcendental and $f_{1} f_{2} \notin \tilde{F}$.

\section{Remarks.}

Remark 1. The functions satisfying all the conditions of Theorem 1 do exist as shown by the following example. Let $\Gamma(z)$ be the Gamma functiuon and $\Psi=\Gamma^{\prime}(z) / \Gamma(z)$. It has been shown in [1] that

$$
\lim _{r \rightarrow \infty} \frac{T(r, \Psi)}{r}=1 \text { and } m(r, \Psi)=O(\log r) .
$$

Therefore the order of $\Psi$ is 1 and

$$
\Delta(\infty, \Psi)=\varlimsup_{r \rightarrow \infty} \frac{m(r, \Psi)}{T(r, \Psi)}=0 .
$$

Let $\Theta=\{z: \arg z=\pi\}$. Then $\omega=1 / 2$. Clearly $\infty$ points of $\Psi$, i.e., the zeros of $\Gamma(z)$, are attacted to $\Theta$. Thus $\Psi$ satisfies all the conditions of Theorem 1 and consequently $\Psi \in \tilde{F}$.

Remark 2. Theorem 1 also improves a result by Gol'dberg [5], where he obtained that $\delta(a, f)=0$ for any number a under the same hypotheses with $b(z)$ being limited to be a constant.

Remark 3. In theorem 1 , the condition " $\lambda>\omega$ " cannot be weakened. In fact, Theorem 1 will be not always valid for meromorphic functions with $\lambda \leqq \omega$. If $\lambda=0$, then $f(z) \equiv z$ will give a counterexample. If $0<\lambda \leqq \omega$, let's consider the system $\Theta=\{z \mid \arg z=\pi\}$. Then in this case, $\omega=1 / 2$. Suppose that $f_{1}(z)$ is an entire function of genus zero, that $f_{1}(z)$ has real negative zeros and $f_{1}(0)=1$. Then we have

$$
\ln f_{1}(z)=z \int_{0}^{\infty} \frac{n(t, 0)}{t(z+t)} d t . \quad(\text { see }[7, \text { p. 117]) }
$$

Suppose that $n(t, 0)=\left[\alpha^{t^{\lambda}}\right]$, where $\alpha \geqq 0$ and $0<\lambda \leqq 1 / 2$. Let $f(z, \alpha, \lambda)=f_{1}(z)$. Assume $\beta \geqq 0$ such that $\beta \leqq \alpha$ and $\alpha \cos \lambda \pi \geqq \beta$ and set $f(z)=f(z, \alpha, \lambda) / f(-z, \beta, \lambda)$. Then by $[7$, p. 117], we will have

$$
m\left(r, \frac{1}{f}\right)=O(\log r), \quad m(r, f)=\frac{\alpha-\beta}{\lambda} r^{\lambda}+O(\log r) \text { and } T(r, f) \sim \frac{\alpha r^{\lambda}}{\lambda} .
$$

Hence $\Delta(0, f)=0, f$ is of finite order $\lambda(0<\lambda \leqq 1 / 2)$. It's clear that $n(r, 0, \Theta, \varepsilon, f)$ $\equiv 0=o\{T(r, f)\}$ by the construction of $f$, i.e., 0-points of $f$ are attracted to $\Theta$. However,

$$
\delta(\infty, f)=\frac{\alpha-\beta}{\alpha} \neq 0
$$




\section{A Further Result.}

In the case when $f$ may be of infinite order, we will have the following result (Theorem 3) in which we will say $T(r, f) \in S_{u}$ if $\max \left\{T(t, f) t^{-u} \mid 1 \leqq t \leqq r\right\}=$ $O\left\{T(r, f) r^{-u}\right\}$ as $(r \rightarrow \infty)(0 \leqq u<\infty)$.

THEOREM 3. Suppose that $\Theta$ is some system of rays and $f$ is a meromorphic function of finite lower order $\lambda>\omega$ satisfying $T(r, f) \in S_{u}$ for some $u>\omega$. If $\Delta(b(z), f)=0$ and $b(z)$-points of $f$ are attracted to $\Theta$ for a small function $b(z)(b(z)$ can be $\infty)$. Then $\delta(a(z), f)=0$ for any small function $a(z)$, including $\infty$. That $\imath s, f \in \tilde{F}$.

Proof. We can assume that $a(z) \neq \equiv, b(z) \not \equiv \infty$ and $\Theta=\{z \mid \arg z=0$ ) (see the proof of theorem 1). Let $m$ be a large positive integer, $n=m^{5}, \alpha \in[1 / n, 2 / n]$, $\beta=2 \pi-\alpha$ and $k=\pi /(\beta-\alpha)=\pi / 2(\pi-\alpha)$. Then by lemma 4 ,

$$
B_{\alpha \beta}(r, f=a(z)) \leqq S_{\alpha \beta}(r, f=b(z))+S_{\alpha \beta}(r, b(z)-a(z))+O(1) r^{k} \quad \text { as } r \rightarrow \infty .
$$

Also by lemma 6 ,

$$
S_{\alpha \beta}(r, b(z)-a(z))=A_{\alpha \beta}(r, b(z)-a(z))+o\{T(r, f)\} \quad \text { as } \quad r \rightarrow \infty .
$$

By using the same method as in the proof of Theorem 1 (see (17)) and in view of the fact $u>\omega$, we can deduce that

$$
S_{\alpha \beta}(r, f=b(z)) \leqq A_{\alpha \beta}(r, f=b(z))+o\{T(r, f)\} .
$$

Hence by (22), (23), (24), we have that

$$
\begin{aligned}
B_{\alpha \beta}(r, f=a(z)) & \leqq A_{\alpha \beta}(r, f=b(z))+A_{\alpha \beta}(r, b(z)-a(z))+o\{T(r, f)\}+O(1) r^{k} \\
& =A_{\alpha \beta}(r, f=b(z))+A_{\alpha \beta}(r, b(z)-a(z))+o\{T(r, f)\},
\end{aligned}
$$

since the lower order $\lambda>k$ for large $n$.

Now using the same arguments as in the proof of Theorem 1, we can obtain that

$$
\int_{4 / n}^{2 \pi-4 / n} \ln +\frac{1}{\left|f\left(r e^{2 \phi}\right)-a\left(r e^{2 \phi}\right)\right|} d \phi=o\left\{T\left(r_{\jmath}, f\right)\right\} .
$$

It's easy to verify that

$$
\varliminf_{r \rightarrow \infty} \frac{\ln T\left(r \cdot \frac{1}{f-a(z)}\right)}{\ln r} \leqq \lambda .
$$

Hence there exists a sequence $\left\{R_{j}\right\}$ such that $R_{j} \rightarrow \infty$ as $\jmath \rightarrow \infty$ and for $R \in$ $\left\{R_{j}\right\}$ we have

$$
T\left(R, \frac{1}{f-a(z)}\right)<R^{\lambda+1}
$$


By lemma 1, we can find a set $E$ with $\ln \operatorname{mes}(E \cap[1, R]) \geqq(1-1 / m) \ln R+O(1)$ as $R \rightarrow \infty$ such that for $r \in E$,

$$
\ln M\left(r, \frac{1}{f-a(z)}\right) \leqq C_{*}(\lambda+1)^{2} m^{4} T(r, f),
$$

where $C_{*}$ is an absolute constant. Therefore,

$$
\begin{aligned}
\int_{-4 / n}^{4 / n} \ln +\frac{1}{\left|f\left(r e^{2 \phi}\right)-a\left(r e^{2 \phi}\right)\right|} d \phi & \leqq \int_{-4 / n}^{4 / n} \ln M\left(r \cdot \frac{1}{f-a(z)}\right) d \phi \\
& \leqq \frac{8}{m^{5}} C_{*}(\lambda+1)^{2} m^{4} T(r, f) \\
& =\frac{8}{m} C_{*}(\lambda+1)^{2} T(r, f) .
\end{aligned}
$$

Combining (26) with (25), for $r \in E$,

$$
m\left(r, \frac{1}{f-a(z)}\right) \leqq o\{T(r, f)\}+\frac{8}{m} C_{*}(\lambda+1)^{2} T(r, f) .
$$

But $m$ can be assumed arbitrarily large. We thus have $\delta(a(z), f)=0$. The proof is completeed.

The authors would like to acknowledge useful suggestions by the referee.

\section{REFERENCES}

[1] S. B. BANK AND R.P. KAUfMAN, An extension of Hölder's theorem concerning the Gamma function, Funkcialaj Elevacioj, 19 (1976), 53-63.

[2] C. T. Chuang, C.C. Yang And H.X. Yi, Meromorphic functions which assume no deficient functions, preprint.

[3] C.T. Chuang, Sur les fonctions-types, Scientia Sinica, 10 (1961), 171-181.

[4] W.H.J. Fuchs, Developments in the Classical Nevanlinna Theory of meromorphic functions, Bull Amer. Math. Soc. 73 (1967), 275-291.

[5] A.A. Gol'DBerg, Distribution of the value of meromorphic functions with poles attracted to a system of rays, Ukrainskii Matematicheskii Zhurnal, vol. 41, no. 6 (1989), 634-638.

[6] A.A. Gol'DBerg AND L.V. Ostrovskit, Theory of distribution of the value of meromorphic functions, Nauka, Moscow (1970).

[7] W.K. HAYMan, Meromorphic functions, Oxford Univ. Press 1964.

[8] Bao QIN LI, Remarks on a result of Hayman, Kodai Math. J. vol. 11, no. 1 (1988), 32-37.

[9] Bao QIN LI, On the quantity $\delta_{5}(g(z), f)$ of gappy entire functions, Kodai Math. J. vol. 11 , no. 2 (1988), 287-294.

[10] Bao Qin Li ANd ChONG Ji DaI, On the modulus distribution of Fabry gap power series, Math. Annals (in Chinese), vol. 10A (5) 1989, 605-612.

[11] L.R. Sons, An analogue of a theorem of W.H.J. Fuchs on gap series, Proc. London Math. Soc. (3), vol, 21, Nov. 1970, 525-539. 
[12] N. Steinmetz, Eine Verallgemeinerung des $z$ weiten Nevanlinnaschen Hauptsatzes, J. Reine Angew. Math., 368 (1986), 134-141.

[13] L. YANG, Value distribution theory and its new research, Academic Press, New York, 1982.

Department of Mathematics

The Hong Kong University of Science and Technology

HONG KONG

AND

Department of Mathematics

UNIVERSITY OF MARYLAND

College Park, MD. 20742

U.S.A. 